\title{
El rol del Estado y su influencia en las condiciones de vida de la mujer desplazada. Medellín, 2015
}

\section{The role of the State and its influence on the living conditions of displaced women. Medellín, 2015}

Recibido: 03 de diciembre de 2015 - Revisado: 02 de junio de 2016 - Aceptado: 24 de junio de 2016.

Isabel Cristina Posada Zapata ${ }^{2}$

Abraham Mendoza Ríos ${ }^{3}$

Sara María Cano Bedoya ${ }^{4}$

Isabel Cristina Orozco Giraldo ${ }^{5}$

Carolina Restrepo Correa ${ }^{6}$

\section{Resumen}

Este artículo se propone presentar la comprensión que han hecho las mujeres en situación de desplazamiento acerca del rol que ha desempeñado el Estado y su influencia en las condiciones de vida de la mujer desplazada. Se parte de un enfoque cualitativo, tomando para el análisis elementos de la teoría fundamentada de Strauss y Corbin. La muestra estuvo compuesta por 49 mujeres en situación de desplazamiento. Para el análisis se utilizó la codificación, categorización e interpretación y finalmente se llevó a cabo la teorización. El rol que desempeña el Estado colombiano influye desde la experiencia del desplazamiento hasta la percepción de salud/enfermedad y la búsqueda de soluciones en salud, ya que este ha dispuesto múltiples leyes para la protección de las mujeres desplazadas, pero pocas veces se han cumplido.

\section{Palabras clave}

Colombia, conflicto armado, violencia, migración humana, salud de la mujer.

\begin{abstract}
This article intends to present the understanding that women in displacement have made about the role played by the State and its influence on the living conditions of displaced women. It starts from a qualitative approach, taking for the analysis elements of the reasoned theory of Strauss and Corbin. The sample consisted of 49 women in displacement situation. For the analysis we used coding, categorization and interpretation and finally theorization was carried out. The role of the Colombian State influences from the experience of displacement to the perception of health/illness and the search for solutions in health, since it has provided multiple laws for the protection of displaced women, but they have rarely been fulfilled.
\end{abstract}

\section{Keywords}

Colombia, armed conflict, violence, human migration, health of the woman.

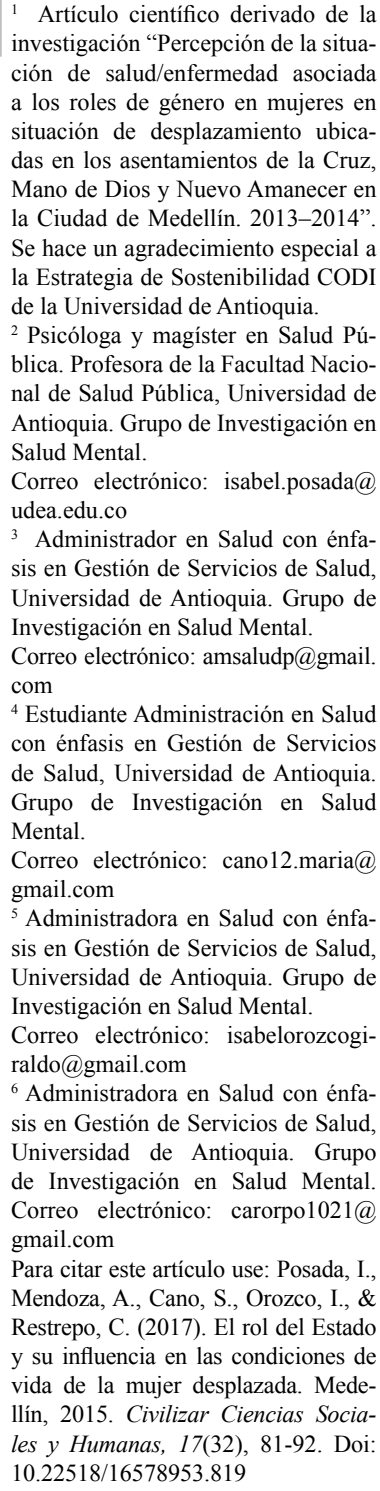

Artículo científico derivado de la investigación "Percepción de la situación de salud/enfermedad asociada a los roles de género en mujeres en situación de desplazamiento ubicadas en los asentamientos de la Cruz, Mano de Dios y Nuevo Amanecer en la Ciudad de Medellín. 2013-2014". Se hace un agradecimiento especial a la Estrategia de Sostenibilidad CODI de la Universidad de Antioquia.

${ }^{2}$ Psicóloga y magíster en Salud Pública. Profesora de la Facultad Nacional de Salud Pública, Universidad de Antioquia. Grupo de Investigación en Salud Mental.

Correo electrónico: isabel.posada@ udea.edu.co

3 Administrador en Salud con énfasis en Gestión de Servicios de Salud, Universidad de Antioquia. Grupo de Investigación en Salud Mental. Correo electrónico: amsaludp@gmail. com

${ }^{4}$ Estudiante Administración en Salud con énfasis en Gestión de Servicios de Salud, Universidad de Antioquia. Grupo de Investigación en Salud Mental.

Correo electrónico: cano12.maria@ gmail.com

${ }^{5}$ Administradora en Salud con énfasis en Gestión de Servicios de Salud, Universidad de Antioquia. Grupo de Investigación en Salud Mental.

Correo electrónico: isabelorozcogiraldo@gmail.com

${ }^{6}$ Administradora en Salud con énfasis en Gestión de Servicios de Salud, Universidad de Antioquia. Grupo de Investigación en Salud Mental. Correo electrónico: carorpo1021@ gmail.com

Para citar este artículo use: Posada, I., Mendoza, A., Cano, S., Orozco, I., \& Restrepo, C. (2017). El rol del Estado y su influencia en las condiciones de vida de la mujer desplazada. Medellín, 2015. Civilizar Ciencias Sociales y Humanas, 17(32), 81-92. Doi: $10.22518 / 16578953.819$ 


\section{Introducción}

Los conflictos armados en el mundo se han caracterizado por los múltiples ataques contra la población civil, la vulneración generalizada de los derechos humanos, las violaciones y delitos sexuales que son utilizados como arma de guerra contra mujeres y niños, así como el desplazamiento forzado de muchas personas. En ese sentido, la mayoría de las violaciones cometidas en las épocas de conflicto son con frecuencia sistemáticas y tienen como objetivo aterrorizar a la población, destrozar a las familias y destruir las comunidades, afectando principalmente las poblaciones más vulnerables (Organización de las Naciones Unidas -ONU, 2013).

Colombia hace más de cincuenta años se halla inmersa en un conflicto armado derivado de factores económicos, políticos e institucionales, sociales y culturales; el cual ha involucrado múltiples actores: las guerrillas de izquierda, los paramilitares de derecha, los narcotraficantes, el gobierno, las fuerzas armadas y la sociedad civil, siendo esta última la más afectada (Yaffe, 2011).

De acuerdo con el Centro Nacional de Memoria Histórica (2015), en Colombia la mayoría de población desplazada son mujeres (3.301.848), lo que indica que aproximadamente el $51 \%$ de las víctimas de desplazamiento forzado son niñas, adolescentes, mujeres adultas y adultas mayores, principalmente de origen campesino y étnico. Además, los informes señalan que un $15 \%$ del total de la población afrocolombiana y el $10 \%$ de la población total indígena en Colombia ha sido desplazada, y además que el $87 \%$ de la población expulsada de sus regiones vivía en el campo. Debe destacarse que la mayoría de las víctimas de violencia sexual en el conflicto armado son mujeres afrodescendientes e indígenas. De acuerdo con el informe Forensis 2014, en los departamentos de Chocó y Cauca -que cuentan con población predominantemente afrodescendiente e indíge- na- el $70 \%$ de las presuntas víctimas de violencia sexual fueron mujeres (Comisión Interamericana de Derechos Humanos-CIDH, 2015).

Es por ello que puede afirmarse que el conflicto armado en el país ha dejado miles de víctimas, entre ellas las mujeres, quienes han sido sometidas a violencia tanto física como sexual por parte de los actores armados legales e ilegales (El Estado y la violencia sexual contra las mujeres en el marco de la violencia sociopolítica en Colombia, 2012), estos hechos llevaron a la Corte Constitucional, máximo organismo jurisdiccional en el país, a constatar que: "la violencia sexual es una práctica habitual, extendida, sistemática e invisible en el contexto del conflicto armado colombiano, así como lo son la explotación y el abuso sexual" (Auto 092 de 2008). El Auto 092 de 2008 tiene como propósito la protección de los derechos fundamentales de las mujeres víctimas del desplazamiento forzado por causa del conflicto armado. Esta resolución judicial representa un fallo histórico, ya que nunca antes en la jurisprudencia nacional una corporación de justicia se había dado a la tarea de analizar la situación de las mujeres como colectivo y frente a un delito (Programa de las Naciones Unidas para el Desarrollo -PNUD, 2010).

Sin embargo, los delitos sexuales no son el único problema, en los conflictos armados la población es sometida a condiciones que rompen el equilibrio psíquico de los sujetos, pues son pocas las posibilidades de detener la acción violenta del otro (Aristizábal \& Palacio, 2003), las mujeres en especial son víctimas de asesinatos, torturas, amenazas, desapariciones forzadas, detenciones ilegales, secuestros $\mathrm{y}$ desplazamientos, estos hechos afectan mucho más a las mujeres indígenas y afrocolombianas acrecentando la discriminación racial y cultural que han vivido a lo largo de la historia (International Center for Transitional Justice ICTJ, 2014). 
Si bien es cierto que el Estado colombiano ha avanzado hacia un mayor reconocimiento público de la violencia sexual contra las mujeres en situación de desplazamiento y ha realizado avances normativos en el tema como respuesta a las alertas hechas por diversas instancias de Naciones Unidas y de la Comisión Interamericana de Derechos Humanos, así como de organizaciones internacionales y nacionales de mujeres y de derechos humanos, también es oportuno decir que el Estado no cuenta con suficiente voluntad política expresada en presupuestos adecuados y en articulaciones institucionales que racionalicen y den operatividad a la oferta institucional, lo que ocasiona una respuesta inadecuada e ineficiente a la problemática (El Estado y la violencia sexual contra las mujeres en el marco de la violencia sociopolítica en Colombia, 2012).

De acuerdo con los planteamientos anteriores, la investigación desarrolló como objetivos específicos describir la percepción de los roles de género asociados a la situación de salud/enfermedad de acuerdo con diferencias en las condiciones sociodemográficas, étnicas y el tipo de desplazamiento que experimentaron las mujeres. En este contexto, este artículo se propone presentar la comprensión que han hecho las mujeres en situación de desplazamiento acerca del rol que ha desempeñado el Estado y su influencia en las condiciones de vida de la mujer desplazada, ya que esta categoría fue una de las más relevantes del estudio.

Esta investigación se enmarca en el ámbito de la Salud Pública, entendiendo su interés por la salud de todas las poblaciones. Asimismo este estudio se fundamenta desde un enfoque de género como determinante de la salud (Organización Panamericana de la Salud-OPS, 2010) porque entiende la relación indisoluble entre esta y las condiciones sociales, relación que ha sido estudiada y que permite entender que las mujeres en los conflictos bélicos se exponen a violencias específicas relacionadas con su género, los imaginarios socioculturales respecto a ellas y su capacidad de resiliencia (Montoya, Romero, \& Jeréz, 2013).

En este contexto la salud y la enfermedad hacen parte de un continuo que puede entenderse como la presencia/ausencia de capacidades superiores para hacer frente a las adversidades del medio (Barros \& Delizoicov, 2008), capacidades que deben vivirse en el marco del desplazamiento forzado, constituido por la huida obligada de miles de familias y comunidades de sus lugares de origen, como acto para salvaguardar sus vidas, constituyendo la crisis humanitaria más dramática en Colombia (Centro Nacional de Memoria Histórica, 2015).

\section{Metodología}

Esta investigación parte de un enfoque hermenéutico como marco epistemológico. Se privilegia una posición horizontal entre el investigador y los participantes del estudio, dando lugar a un acto comunicativo que busca la interpretación de los discursos y la comprensión de una realidad social (Habermas, 1986). Utilizando el interaccionismo simbólico como fundamento teórico, se analizaron los significados de los actos y las palabras para estas personas, y la forma como estos se construyen en la interacción con otros, para finalmente agruparlos, y constituir un conjunto de sentidos alrededor del discurso de estas mujeres (Blumer, 1981).

Para esta investigación se emplearon las herramientas propuestas por el método de Teoría Fundada, la cual permite hacer un acercamiento a la comprensión del conocimiento que tienen los sujetos y así lograr aproximaciones sistemáticas para descubrir aspectos significativos de la experiencia humana que permanecen inaccesibles a través de los métodos de verificación tradicionales. En este método, la recolección de datos, el análisis y la teoría que surgió de ellos guardan estrecha relación entre sí 
(Strauss \& Corbin, 2002). Consecuentemente, se buscó hacer una interpretación de los conceptos ligados a las experiencias de estas mujeres y se desarrolló una categorización de los datos, guardando los criterios de calidad en la investigación cualitativa como la credibilidad (reconocimiento de la validez), la auditabilidad (búsqueda de la neutralidad de la interpretación) y la transferibilidad (posibilidad de llevar los resultados a contextos similares) (Castillo \& Vásquez, 2003).

La población fue constituida por mujeres mayores de edad, que hubieran vivido la condición de desplazamiento durante al menos un año, y que para el momento de la investigación, habitaran en alguno de los tres asentamientos seleccionados. Se realizaron 15 entrevistas, 6 grupos focales y 6 entrevistas a profundidad, con una participación total de 49 mujeres. En las entrevistas se trataron temas como la experiencia del desplazamiento, la relación entre los roles que desempeña con su estado de salud y la percepción de su familia; en los grupos focales se habló de cómo las mujeres aportaban al cuidado de su salud y de sus familias, cómo había cambiado el cuidado de su salud luego del desplazamiento y en la relación de las condiciones sociales con la enfermedad; finalmente, las entrevistas a profundidad se realizaron con el fin de poner a prueba las hipótesis que se habían construido durante toda la investigación. Para la selección de las participantes se utilizó el muestreo teórico, lo que implicó elegir la población de acuerdo con las necesidades de las categorías.

Estas mujeres fueron contactadas en tres asentamientos en la ciudad de Medellín. El asentamiento La Cruz está ubicado al extremo nororiental de la ciudad y hace parte de la comuna 3 (Manrique), allí la población en su mayoría es desplazada. Las condiciones sociales de esta población son de alta vulnerabilidad y sus ocupaciones laborales se concentran en la construcción, los oficios varios y la informalidad (Ortiz, 2013).

Mano de Dios era un asentamiento que se encontraba cerca al centro de Medellín, zona receptora de familias desplazadas, en su mayoría afrodescendientes. En el año 2003 en este asentamiento ocurrió un incendio que dejó muchos damnificados y como consecuencia estas familias sufrieron un desplazamiento interno en la ciudad, lo que significó un doble desplazamiento. Estas personas fueron reubicadas en el barrio Nuevo Amanecer, en el corregimiento Belén Altavista, conformado por 3.600 personas aproximadamente, quienes igualmente presentan condiciones sociales precarias; si bien se intentó que en la muestra participaran mujeres indígenas, que se ha demostrado también han sufrido la experiencia del desplazamiento (Unidad para las Víctimas, 2015), no fue posible contactar en los asentamientos a mujeres de esta etnia, quedando conformada la muestra solo por mujeres afro y mestizas.

Se tuvieron en cuenta fuentes de información primarias, constituidas por los discursos de las participantes; y secundarias, compuestas por la revisión de documentos oficiales, literatura y otras investigaciones.

El plan de análisis consistió en tres procedimientos básicos: la codificación, a través de un análisis línea por línea de las entrevistas realizadas con las participantes, iniciando con una codificación abierta, pasando a una analítica que busca relaciones y, finalmente, llegando a una selectiva que busca fortalecer la interpretación; la categorización, donde se realizó una agrupación posterior en conjuntos que respondían desde lo descriptivo, lo analítico y lo interpretativo; y, finalmente, se llevó a cabo la teorización, es decir, la construcción de un modelo explicativo que respondiera a la pregunta de investigación (Strauss \& Corbin, 2002). 
Grafico 1.

Principales categorías

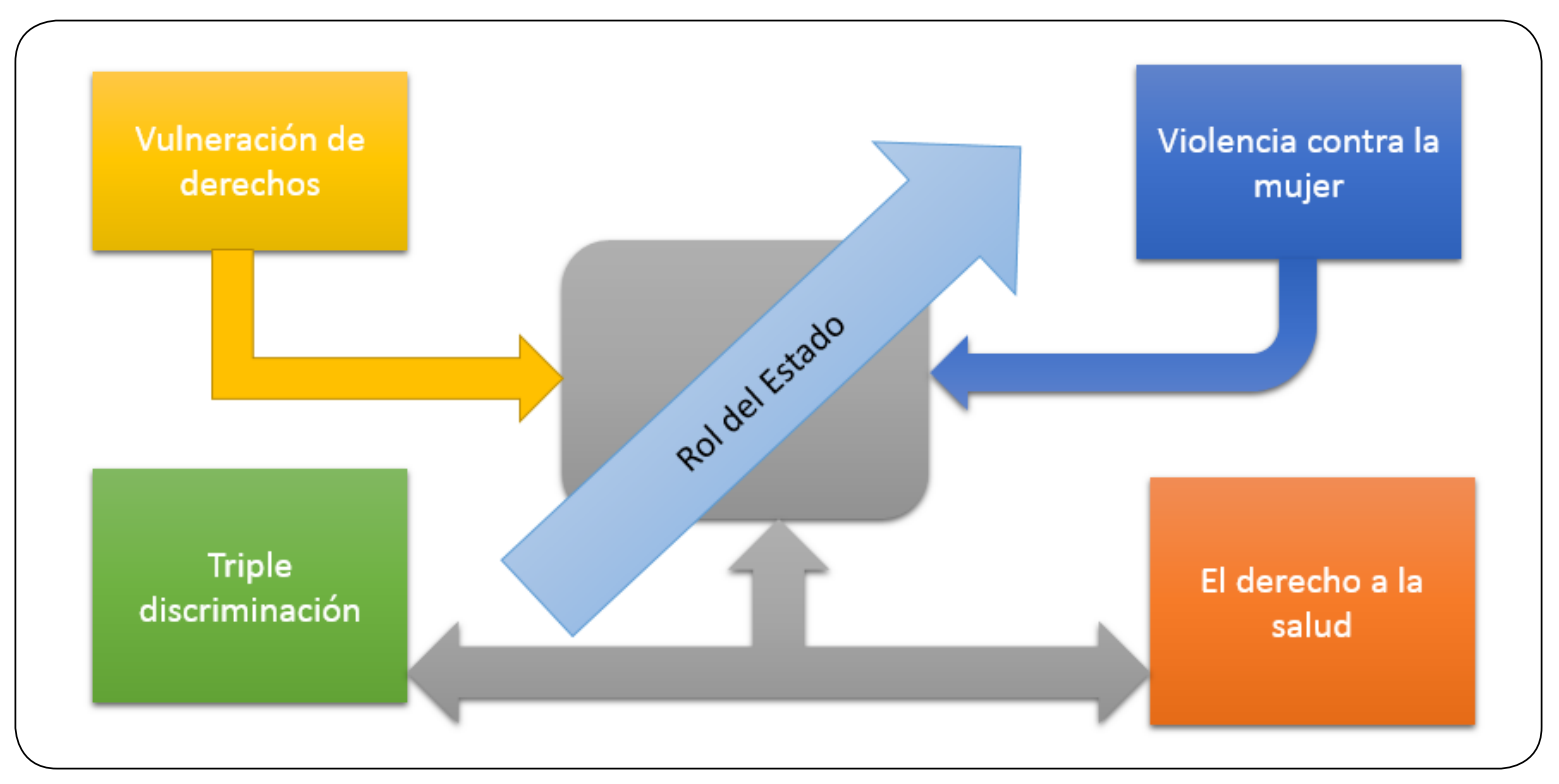

Fuente: elaboración propia.

Luego del análisis, se realizó la validación de los hallazgos por medio de una socialización ante la comunidad en general de los asentamientos, donde las participantes reconocieron la validez de los resultados expuestos.

Para la ejecución de esta investigación se tuvieron en cuenta los criterios establecidos en la Declaración de Helsinki, donde se insta a los investigadores a garantizar la protección de la vida, la salud, la dignidad, la integridad, a la libre determinación, privacidad y confidencialidad de la información personal de la población sujeto (Asamblea Médica Mundial- AMM, 1964). Así mismo, se tuvo en cuenta la Resolución 8430 de 1993 del Ministerio de Salud de Colombia que regula la investigación con seres humanos, y desde allí este proyecto fue catalogado como de riesgo mayor al mínimo, por lo que siempre se estuvo atento a los posibles riesgos psicosociales que se derivaran del discurso de las participantes. Todas las participantes en el estudio tuvieron la posibilidad de firmar un formato de consentimiento informado en el cual se les garantizó la privacidad de la información proporcionada. Teniendo en cuenta estas con- sideraciones, se obtuvo el aval del Comité de Ética de la Facultad Nacional de Salud Pública de la Universidad de Antioquia.

\section{Resultados y discusión}

Para dar respuesta a la pregunta de investigación formulada es preciso señalar que el Estado colombiano tiene uno de los marcos legales "más avanzados del mundo" (Peace Brigades International Colombia, 2010), sin embargo, varias de las sentencias de la Corte Constitucional colombiana muestran que las instituciones estatales siguen desatendiendo las necesidades de la población civil desplazada o amenazada por desplazamiento (PNUD, 2010). Esta situación expresada por la Corte, es confirmada por una mujer participante:

"Yo me acuerdo que nosotros fuimos a poner tres denuncias a la Fiscalía por las amenazas que estábamos viviendo y que a uno le tenga que decir que: -si tiene para dónde irse que se vaya-, pues es increíble que una persona que sea un líder comunitario, que le está brindando pues su labor a la sociedad, el Estado le tenga que decir eso. Para mí eso es muy duro. 
No quería llorar y termine llorando" (Grupo focal 1, código 144, comunicación personal, mayo de 2014).

En la Constitución Política de Colombia de 1991, en el artículo segundo, se establece que:

Las autoridades de la República están instituidas para proteger a todas las personas residentes en Colombia, en su vida, honra y bienes, creencias y demás derechos y libertades con el fin de asegurar el cumplimiento de los deberes sociales del Estado y los particulares.

Sin embargo, una de las investigaciones consultadas, sobre la responsabilidad del Estado en el fenómeno del desplazamiento, resaltó la violación de las normas establecidas por este, respecto a obligaciones y deberes contenidos en textos específicos como las leyes, reglamentos o estatutos que establecen las formas en las que debe protegerse a la población desplazada (Salamanca, 2010).

Por esta razón al indagar en las mujeres participantes de esta investigación sobre el rol del Estado colombiano en su situación, ellas consideran que fueron abandonadas durante todo el proceso del desplazamiento ya que no contaron con la protección de las instituciones gubernamentales para afrontar tal contingencia:

"Todo el mundo andaba amenazado entonces... imagínese que para recoger el cadáver de mi casa, eh... nos tocó... a mi hermana le tocó recogerlo sola y llevarlo sola pal' pueblo, [...] imagínese que me tocó acompañarla a ella a la morgue a llevarlo, sacarlo del ataúd, esperar que le hicieran la necropsia con ella solita, esperar que pa' metelo otra vez a la caja y nadie se nos arrimó [...]. Entonces mire que eso es muy duro, eso son cosas muy crueles" (Grupo focal 1, código 152, comunicación personal, mayo de 2014).

Además de la ausencia del Estado en las distintas situaciones vividas de las mujeres desplazadas, estas piensan que una de las causas del desplazamiento está relacionada con la res- puesta institucional que se ha presentado para el conflicto armado en Colombia, lo cual ocasiona un escenario propicio para el fenómeno del desplazamiento:

"Porque si el Estado manejara bien esos conflictos armados, manejara bien todo eso, que cambiaran, que un proceso de paz que nunca ha llegado, que cada día es más fuerte, entonces ¿por qué es eso? Por el Gobierno, todas esas cosas son por el mismo Gobierno" (Entrevista a profundidad 2, código 52, comunicación personal, junio de 2014).

En ese contexto, las mujeres desplazadas encuentran una responsabilidad en la pasividad del Estado, que no ha intervenido adecuadamente en el fenómeno de la violencia en Colombia, lo que les afecta al llegar a una nueva ciudad, pues ello no significa necesariamente que el desplazamiento termine ni que la persona desplazada ya no esté involucrada en el conflicto.

En Colombia existen diversas causas de desplazamiento forzado, de acuerdo con Segura (2001), hay tres tipos distintos de migración forzada como son las relacionadas con la economía (falta de dinero, desempleo), ambientales (inundaciones, derrumbes, terremotos) y el desplazamiento por razones de violencia. Esta última es la principal causa del desplazamiento que se vive en Colombia, el cual se ha caracterizado en los últimos años por las graves violaciones a los Derechos Humanos (DDHH) y al Derecho Internacional Humanitario (Arias, 2002).

Un estudio realizado por Quevedo (2013) documentó el tipo de estrategias utilizadas en el conflicto armado colombiano contra las mujeres que incluyen agresiones de naturaleza física, psicológica y sexual, teniendo por objetivo el lesionar, aterrorizar y debilitar al enemigo para avanzar en el control de territorios y los recursos económicos. En este orden de ideas puede entenderse cómo las mujeres son desplazadas de sus lugares de origen a causa del conflicto 
armado, lo que genera que ellas lleguen a nuevas comunidades. Al arribar a este nuevo lugar, las mujeres llegan desorientadas y confundidas debido al cambio en la dinámica de los papeles familiares ante la muerte o pérdida del esposo o compañero, el trauma físico y psicológico producido por hechos de violencia y las amenazas padecidas (Comisión Interamericana de Derechos Humanos, CIDH, 2006). Sumado a lo anterior, la necesidad de adaptarse social y económicamente a una nueva comunidad lleva a percibir de manera diferente y sentir de manera distinta los efectos producidos por la violencia.

Para entender las condiciones ligadas a la situación crítica de la llegada a la ciudad como población desplazada, es preciso señalar que al llegar a Medellín las mujeres buscan reconstruir sus vidas, sin embargo, se ven expuestas a conflictos comunitarios en el asentamiento, lo que de alguna manera puede incidir en el aumento de su vulnerabilidad. No obstante, algunas participantes de la investigación expresaron que la violencia generada en los pueblos es más fuerte que la que se vive en la ciudad, que, según ellas, solo involucra a algunos participantes:

"Pues la diferencia era que allá pues iban directamente las personas donde uno estaba, nosotros no teníamos problemas con nadie, pero allá había muchos grupos [...] y acá no, acá pues ellos tienen sus problemas con quien los tienen, y nosotros no tenemos nada que ver" (Grupo focal 3, código 136, comunicación personal, mayo de 2014).

Sumado a lo anterior, también algunas mujeres expresaron que la violencia en los pueblos es más cruda:

"Yo de verdad levanté los hijos míos en medio de la violencia, desde muy pequeñitos porque en el pueblo es muy fuerte la violencia porque imagínese, entrar la guerrilla [...] detonan unas cosas como unas granadas pero muy fuertes, que eso se estremece todo donde una está [...] Vea, son unas bombas, no son granadas, son bombas y granadas, y eso es un pañito, lo de por aquí, son pañitos de agua, las personas que no han tenido pues como esa experiencia de sentir cuando entra esa gente al pueblo, eso es horrible" (Grupo focal 1, código 120, comunicación personal, mayo de 2014).

De igual manera, las mujeres desplazadas deben enfrentarse a la marginación y rechazo social en la ciudad sufriendo triple discriminación, en primer lugar, por el hecho de ser mujeres, en segundo lugar, por el origen étnico y, finalmente, por el hecho en sí de ser desplazadas.

En primer lugar, la mayoría de mujeres desplazadas han sido educadas bajo la separación rígida de los roles masculinos y femeninos, las actividades que desarrollaban antes de estar condicionadas por el desplazamiento giraban en torno al hogar y la maternidad, todo lo anterior bajo el mandato masculino (Arias, 2002), asignándoles la posición de dependencia, pero el impacto producido por la violencia del conflicto armado y posterior desplazamiento a la ciudad, obliga a las mujeres a reconfigurar los roles que socialmente se les estaban asignados, convirtiéndose en jefes de hogar o en apoyo a sus parejas en la búsqueda del sustento de la familia, sin embargo, algunas entrevistadas expresaron lo difícil que ha sido para ellas este nuevo papel, ya que sus parejas desean que nuevamente la mujer se dedique a las tareas del hogar:

"En el caso mío al esposo mío tampoco le gusta que yo trabaje, él en este momento porque le toca, pero, él quisiera yo no me moviera de la casa, pues la verdad es que yo no lo entiendo, no sé qué le pasa o por qué, o sea, si son celos o es egoísmo, o qué" (Grupo focal 3 , código 104, comunicación personal, mayo de 2014).

En segundo lugar, el desplazamiento en Colombia ha afectado profundamente a las minorías étnicas, especialmente a la población de mujeres afrocolombianas, que son despojadas de su identidad, cultura y territorialidad por el conflicto armado. Al llegar a los nuevos lugares de asentamiento en la ciudad, las mujeres desplazadas afrocolombianas son discriminadas 
por parte de algunos habitantes (CIDH, 2015), así fue expresado por varias entrevistadas:

"A un señor le dije un día: -Córtese usted y me corto yo, qué nos corre, qué nos sale, qué le sale a usted que es blanco y qué me sale a mí que soy negra-, y sabe qué me contestó: 'la sangre suya sale negra y la mía sale roja"' (Grupo focal 3, código 146, comunicación personal, mayo de 2014).

En síntesis, las mujeres desplazadas afrocolombianas padecen una alta vulnerabilidad socioeconómica con respecto al resto de la población que está condicionada por el desplazamiento. De igual forma, las mujeres desplazadas en la ciudad deben enfrentarse a la marginación y rechazo social originado por las comunidades donde comienzan a residir, debido a la percepción que tienen algunos habitantes de ser extrañas o desconocidas en la comunidad, esto puede llevar a que estos ciudadanos se tornan indiferentes ante el fenómeno del desplazamiento o por el contrario se vuelvan hostiles ante el temor de lo desconocido, así fue manifestado por una entrevistada:

"Incluso cuando una vez que salí yo a pedir con mi niño chiquito a mí me sacaron un cuchillo [...] pensaron que yo iba a robar, y yo no le robo a nadie" (Grupo focal 5, código 246, comunicación personal, mayo de 2014).

El impacto del desplazamiento y las nuevas condiciones de vida en el lugar de asentamiento en la ciudad afectan el estado de salud de la población desplazada, especialmente a las mujeres que están sujetas a dicha condición de vulnerabilidad, sin embargo, al tratar de acceder a los servicios de salud las mujeres desplazadas se sienten discriminadas al tener dificultades para ser atendidas en los servicios asistenciales o, por el contrario, se sienten atropelladas por la atención recibida allí, así fue expresado por algunas de las entrevistadas:

"Mi mamá estaba enferma, entonces comencé a insultar al vigilante porque no la atendía y me dice: 'es que esta negra'. Así me dijo: ¡negra!” (Grupo focal 6, código 82, comunicación personal, mayo de 2014).

En concordancia con lo anterior, con el desplazamiento las mujeres pierden el acceso a los servicios de salud a los que habitualmente acudían, adicionalmente, tienen desventajas culturales, económicas y funcionales en los servicios de salud en donde se reubican (Ballesteros, Gaviria, \& Martínez, 2006). Por estas razones las mujeres en condición de desplazamiento esperan que el Estado tenga un rol mucho más participativo en la búsqueda de soluciones en salud para la población desplazada de mayor vulnerabilidad, sin embargo, se encuentran escépticas tras las dificultades en la atención en salud y solo acuden a los servicios cuando están condicionadas por una enfermedad:

"Porque es que mire que si el Gobierno fuera diferente o pusiera más médicos, él dijo que prioridad para los más pobres, fuera como mejor, ¿no? Porque es que uno pa' una cita médica, eso es misión imposible" (Grupo focal 5, código 82, comunicación personal, mayo de 2014).

Ante las necesidades referidas por las mujeres desplazadas en el acceso a los servicios de salud, algunas de las entrevistadas manifestaron conocer sus derechos en salud, pero que el Estado no se los respeta, es por ello por lo que recurren como última instancia a la utilización de la tutela como herramienta jurídica que ayude a la preservación del derecho a la salud:

"Si uno no entutela, no, no hay operaciones, no hay medicamentos, no hay nada $[. .$.$] por-$ que el Estado dice ¡no!, entonces qué hay que hacer, cuando vemos que estamos acorralados, buscamos un apoyo, por eso buscamos hacer la tutela" (Entrevista a profundidad, códigos 108, 109 y 110, comunicación personal, mayo de 2014).

En conclusión, el rol del Estado colombiano influye desde la experiencia del despla- 
zamiento hasta la percepción de salud/enfermedad y la búsqueda de soluciones en salud, ya que este dispone de múltiples leyes para la protección de la población desplazada. Sin embargo, estas no se han estado cumpliendo a cabalidad, lo que lleva a pensar en una reformulación de acciones orientadas a proteger la vida de las mujeres que nunca pidieron ser desplazadas por su voluntad.

\section{Limitaciones del estudio}

Dado que es un estudio cualitativo, los resultados obtenidos no pueden generalizarse a toda la población de mujeres que han sido desplazadas. Sin embargo, estos resultados permitieron encontrar pistas clave para la comprensión del rol que desempeña el Estado en su experiencia y las acciones que influyen en su calidad de vida y condiciones de salud.

\section{Conclusiones}

Los resultados de esta investigación aportan al direccionamiento de acciones que promuevan una salud integral con un enfoque de género y donde se tenga en cuenta la triple vulnerabilidad de esta población: por ser mujeres, por ser desplazadas y por pertenecer en su gran mayoría a etnias que han presentado históricamente dificultades ligadas a su condición.

El Estado como garante de los derechos humanos debe hacerse presente en aquellos procesos de violencia que afectan a los grupos más vulnerables, entre ellos las mujeres, es decir, hacer cumplir los mecanismos de protección a sus derechos y garantizar una vida digna libre de situaciones adversas que afecten su proceso vital humano.

Las mujeres desplazadas consideran que la vida en la ciudad es muy compleja debido a que al llegar a un entorno urbano, se genera discriminación racial, marginación, choques emocionales y desajustes en el comportamiento, causados también por no contar con un trabajo que provea condiciones mínimas de vida digna.

El Estado colombiano cuenta con los mecanismos legales suficientes para la protección de los derechos fundamentales de las mujeres en situación de desplazamiento. Sin embargo, no están cumpliendo con su objetivo, ya que existe una falta de voluntad política y protagonismo del Estado en el conflicto armado, causando más vulneración a las personas víctimas de este.

Las mujeres desplazadas manifiestan abandono por parte del Estado colombiano luego del fenómeno del desplazamiento, ya que no les brinda las garantías suficientes para desarrollar una vida adecuada en términos de bienestar.

A partir de esta investigación se puede recomendar que para estudios posteriores se aborde la perspectiva de actores gubernamentales que atienden el problema del desplazamiento, para que participen con la población afectada en el diseño de nuevas propuestas integrales para atender y dar respuesta a este fenómeno.

La atención al problema del desplazamiento debe incluir la perspectiva de género, y debe abordar los problemas de vivienda, educación, empleo y todos aquellos de orden psicosocial que afectan la salud de las mujeres.

\section{Referencias}

Asamblea Médica Mundial (1964). Declaración de Helsinki de la AMM -Principios éticos para las investigaciones médicas en seres humanos. Recuperado de: http:// www.isciii.es/ISCIII/es/contenidos/fd-investigacion/fd-evaluacion/fd-evaluacionetica-investigacion/Declaracion-Helsinki-2013-Esp.pdf

Arias, S. (2002). Análisis de género de la situación de salud de la población desplazada y no desplazada en cuatro asentamientos 
marginales de Medellín, 2002. Medellín: Universidad de Antioquia.

Aristizábal, E., \& Palacio, J. (2003). Subjetivación del acto de desplazarse y aspectos psicopatológicos relacionados con experiencias violentas. Investigación y Desarrollo, 11(2), 238-253.

Auto 092 (2008, abril 14). Adopción de medidas para la protección a mujeres víctimas del desplazamiento forzado por causa del conflicto armado. M.P. Manuel José Cepeda Espinosa. Corte Constitucional.

Ballesteros, P., Gaviria, M., \& Martínez, S. (2006). Caracterización del acceso a los servicios de salud en población infantil desplazada y receptora en asentamientos marginales en seis ciudades de Colombia, 2002-2003. Revista Facultad Nacional de Salud Pública, 24(1), 7-17.

Barros, W., \& Delizoicov, D. (2008). Reflexiones epistemológicas en las Ciencias de la salud. Humanidades Médicas, 8(2-3). Recuperado de http://scielo.sld.cu/scielo. php?script $=$ sci arttext\&pid $=\mathrm{S} 1727$ -

$81202008000200001 \& \operatorname{lng}=\mathrm{es} \& \operatorname{tlng}=\mathrm{es}$

Blumer, H. (1981). El interaccionismo simbólico: perspectiva y método. Barcelona: Hora.

Castillo, E., \& Vásquez, M. (2003). El rigor metodológico en la investigación cualitativa. Colombia Médica, 34(3), 164-167.

Centro Nacional de Memoria Histórica. (2015). Una nación desplazada. Informe nacional del desplazamiento forzado en Colombia. Bogotá: Imprenta Nacional de Colombia.

Comisión Interamericana de Derechos Humanos -[CIDH]. (2006). Las mujeres frente a la violencia y la discriminación derivadas del conflicto armado en Colombia. Recu- perado de http://www.cidh.org/countryrep/ ColombiaMujeres06sp/Informe\%20Mujeres\%20Colombia\%202006\%20Espanol. pdf

Comisión Interamericana de Derechos Humanos -[CIDH]. (2015). Informe Anual de la Comisión Interamericana de Derechos Humanos 2015. Capitulo V Colombia. Recuperado de http://www.oas.org/es/ cidh/docs/anual/2015/doc-es/InformeAnual2015-cap5-Colombia-ES.pdf

Constitución Política (1991). Congreso de la República de Colombia. Colombia.

El Estado y la violencia sexual contra las mujeres en el marco de la violencia sociopolítica en Colombia. (2012). Recuperado de http://www.sismamujer.org/ wp-content/uploads/2016/12/39.-Informedel-Estado-y-la-Violencia-Sexual-contralas-Mujeres-en-el-Marco-de-la-ViolenciaSociopol\%C3\%ADtica-en-Colombia.pdf

Habermas, J. (1986). Ciencia y técnica como ideología. Madrid: Tecnos.

International Center for Transitional Justice [ICTJ]. (2014). Las mujeres colombianas afectadas por el conflicto armado son también agentes de cambio. Recuperado de https://www.ictj.org/es/news/mujerescolombianas-afectadas-conflictoarmado-agentes-cambio

Montoya, S., Romero, M., \& Jeréz, L. (2013). Mujer y desplazamiento de sí: sustratos socioculturales que soportan las redes de la violencia. Revista Facultad Nacional de Salud Pública, 31(2), 349-358.

Organización de las Naciones Unidas -[ONU]. (2013). Los conflictos armados. Recuperado de http://www.un.org/es/humanitarian/overview/conflict.shtml 
Organización Panamericana de la Salud [OPS]. (2010). Género y Salud. Una guía práctica para la incorporación de la perspectiva de género en Salud. Recuperado de http://www.paho.org/hq/dmdocuments/2010/manualFinal.pdf?ua $=1$

Ortiz, A. (2013). Caracterización comuna 3 Manrique de la ciudad de Medellín. Recuperado de http://www.kavilando.org/images/stories/documentos/CARACTERIZACION_COMUNA3_MANRIQUE_O_G. pdf

Peace Brigades International Colombia. (2010). Desplazamiento forzado en Colombia. Crimen y tragedia humanitaria. Recuperado de http://www.pbi-colombia.org/ fileadmin/user_files/projects/colombia/files/colomPBIa/100107_boletin_PBI_desplazamiento_2010_WEB.pdf

Programa de las Naciones Unidas para el Desarrollo -[PNUD]. (2010). Las mujeres y la construcción de la paz. Hechos del Callejón, 51. Recuperado de http://www. acnur.org/t3/uploads/media/COI_2710. pdf

Quevedo, H. (2013). Enfoque diferencial y de género para la protección de mujeres victimas de violencia sexual de hechos ocurridos en el contexto de las acciones de los grupos armados que son postulados a la aplicación de Ley 975 de 2005 -de Justicia y Paz (Tesis de maestría). Universidad Nacional de Colombia, Bogotá, Colombia.

Resolución 8430 de 1993. Por la cual se establecen las normas científicas, técnicas y administrativas para la investigación en salud. Ministerio de Salud, octubre de 1993.

Salamanca, K. (2010). Responsabilidad del Estado colombiano por el desplazamiento forzado (Tesis de pregrado). Corporación Universitaria Rafael Núñez, Barranquilla, Colombia.

Segura, N. (2001). Guerra y desplazamiento. Análisis Político, 43, 83-103.

Strauss, A., \& Corbin, J. (2002). Bases de la investigación cualitativa técnicas y procedimientos para desarrollar la teoría fundamentada. Medellín: Universidad de Antioquia.

Unidad para las Víctimas. (2015). Mujeres $y$ conflicto armado. Recuperado de: http://www.unidadvictimas.gov.co/sites/ default/files/documentosbiblioteca/ mujeres.PDF

Yaffe, L. (2011). Conflicto armado en Colombia: análisis de las causas económicas, sociales e institucionales de la oposición violenta. Revista en Ciencias Sociales, 8, 187-208. 
